\title{
Key Design Elements of a Radio-Acoustic-Sounding System for Industrial Applications
}

\author{
Dominik Exel $^{1}$, Stefan Schuster ${ }^{2}$, Stefan Scheiblhofer ${ }^{2}$, Dominik Zankl ${ }^{2}$, Vera Ganglberger ${ }^{2}$, Johann \\ Reisinger ${ }^{2}$, Bernhard G. Zagar ${ }^{1}$ \\ 1 Johannes Kepler University Linz, Institute for Measurement Technology \\ Altenbergerstraße 69, A-4040 Linz \\ ${ }^{2}$ voestalpine Stahl GmbH \\ voestalpine-Straße 3, A-4031 Linz
}

\begin{abstract}
This article discusses compact radio-acoustic-sounding systems (RASSs) for industrial applications. The electromagnetic-acoustic interaction enables to measure temperatures of gases without contact and spatially resolved. For this purpose, the speed of a modulated sound pulse, travelling through the gas with a velocity proportional to the temperature, is determined with a Doppler radar. The optimum sound pulse's in terms of its frequency is discussed. The so called Bragg condition is essential to obtain sufficient receive power. The two most important conditions for an optimal Bragg reflection are the tuning of the wavelengths and an optimal collocation of the sources. These conditions are calculated numerically and verified with real-world measurement data. One purpose of this paper is to summarize physical effects that are key elements to the function of the RASS.
\end{abstract}

Keywords: Radio-Acoustic-Sounding System, RASS, Doppler radar

\section{Introduction}

Non-contact, continuous measurement methods for determining the temperature of gases are used to automate industrial processes. There are many requirements for an universally applicable temperature measurement system. For example, the functional guarantee in a dusty, smoky, and aggressive industrial air, accuracy, safety and last but not least a costeffective realization. The idea of the radioacoustic-sounding system (RASS), which is known from the meteorology [1], is to measure the speed of sound by Doppler radar. The measurement method is based on the effect that an acoustic wave modulates the density of the air. When an electromagnetic wave passes through the air, in which these acoustically induced changes in density occur, a small fraction of the signal will be reflected at each of the transitions. Utilizing the Bragg effect the signal return power can be improved. Therefore the Bragg condition must be fulfilled

$$
\lambda_{e}=2 \cdot \lambda_{a}
$$

with $\lambda_{e}$ the radar wavelength and $\lambda_{a}$ the acoustic wavelength. This leads to a constructive interference and afterwards to a measurable signal power [2, 3].

Due to the fact that the pulsed acoustic wave (red drawn in figure 1), which moves with respect to the source and thus enables spatially resolved measurements, represents the Braggreflector, the backscattered radar signal (black drawn in figure 1) exhibits a Doppler-shift $[4,5]$

$$
f_{\mathrm{d}}=\frac{2\left(c_{\mathrm{a}}+v_{\mathrm{w}}\right)}{c_{\mathrm{e}}} f_{\mathrm{e}}
$$

$c_{\mathrm{e}}$ describes the speed of light $\left(2997 * 10^{5} \frac{\mathrm{m}}{\mathrm{s}}\right)$, and $f_{\mathrm{e}}$ the transmit frequency of the radar. This Doppler-shift $f_{\mathrm{d}}$ is proportional to speed of sound $c_{\mathrm{a}}$ and speed of wind $v_{\mathrm{w}}$ in the propagation direction of the electromagnetic wave. To determine the temperature with this system, the relationship between speed of sound and temperature must be known and will be discussed in Section 2. 

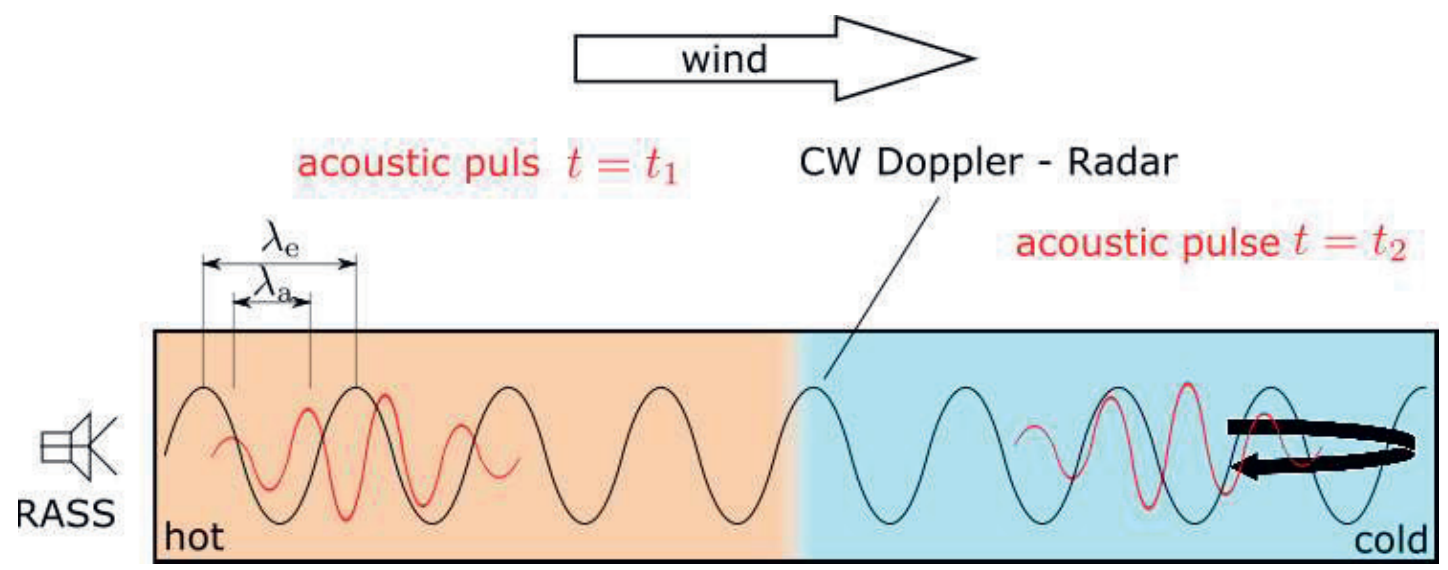

Figure 1: The speed of a transmitted sound pulse is composed of the speed of sound and a wind speed and is measured by a Doppler radar. The acoustic pulse travels through the gaseous medium of interest. By measuring its velocity, spatial temperature measurement is possible.

In industrial applications, the reliability requirements of a measuring system are very high. It is therefore beneficial to characterizes the limits of a system, as it has been done for remote temperature measurement with multispectral camera $[6,7,8]$. Thus it is important to know the backscattering efficiency and this is discussed in Section 3. Section 4 describes the measurement setup with a level plan. Furthermore, the importance of optimal collocation is highlighted. Measurement results of the physical effects are presented and discussed in Section 5.

\section{Speed of sound - temperature relation- ship}

The wave velocity $c_{\mathrm{a}}$ is calculated with the rate of change of the pressure according to the density at atmospheric pressure. Feynman describes it in the derivation of the wave equation for the sound [9]

$$
c_{\mathrm{a}}^{2}=\left(\frac{\mathrm{d} P}{\mathrm{~d} \rho}\right)_{0},
$$

with the pressure $P$ in $\mathrm{Pa}$ and density $\rho$ in $\frac{\mathrm{kg}}{\mathrm{m}^{3}}$. The adiabatic connection between $P$ and $\rho$ is

$$
P=\text { const }: \rho^{\kappa},
$$

with the adiabatic exponent $\kappa$. The derivative from (4) to $\rho$ is

$$
\frac{\mathrm{d} P}{\mathrm{~d} \rho}=\frac{\kappa P}{\rho} .
$$

Combining (3) with (5) leads to

$$
c_{\mathrm{a}}^{2}=\frac{\kappa P}{\rho} .
$$

By extension in the numerator and denominator with the volume $V$, the relations $P V=N k T$ and $\rho V=\mu$ can be used

$$
c_{\mathrm{a}}^{2}=\frac{\kappa N k T}{\mu} .
$$

$T$ describes the temperature in $\mathrm{K}, N$ the Avogadro-constant $\left(6.0221 * 10^{23} \mathrm{~mol}^{-1}\right), \quad k$ the Boltzmann-constant $\left(1.3806 * 10^{-23} \frac{\mathrm{J}}{\mathrm{K}}\right)$ and $\mu$ the molecular mass in $\frac{\mathrm{kg}}{\mathrm{mol}}$. Further, the relation $R_{u}=N k$ can be used and leads to the known formula for the speed of sound

$$
c_{\mathrm{a}}^{2}=\frac{\kappa R_{\mathrm{u}} T}{\mu} .
$$

$\kappa=1.402$ and $\mu=0.02896 \frac{\mathrm{kg}}{\mathrm{mol}}$ can be used for dry air and thus there is a clear correlation between temperature and sound velocity [1]

$$
c_{\mathrm{a}}=20.045 \sqrt{T} \text {. }
$$

\subsection{Speed of sound of a gas mixture}

In industrial applications, it is often necessary to determine the temperature of gas mixtures. For this purpose it is necessary to know the speed of sound for a mixture

$$
c_{\mathrm{a} \_ \text {mix }}=\sqrt{\frac{\left(\sum_{i}^{M} x_{i} \gamma_{i}\right) R_{\mathrm{u}} T}{\left(\sum_{i}^{M} x_{i} \mu_{i}\right)}},
$$

with $M$ components. $x_{i}$ describes the fraction of the $i$ component and $\gamma_{i}$ the molecular heat capacity [10].

\section{RASS equation}

\subsection{Fluctuation of the refractive index}

To be able to calculate the received power, it must be known how much the electromagnetic waves are scattered by the artificial radar target generated by the modulated acoustic 
pulse. Therefore the fluctuation of the refractive index is investigated [11].

Balsley and Gage [12] describes in their work a term for the refractive index $n$

$$
\begin{gathered}
n=1+7.76 * 10^{-7} \frac{p_{\mathrm{a}}}{T}+ \\
+3.73 * 10^{-3} \frac{e}{T^{2}}-40.3 \frac{N_{\mathrm{e}}}{f^{2}},
\end{gathered}
$$

for microwave frequencies in the troposphere. $p_{\mathrm{a}}$ describes the atmospheric pressure in $\mathrm{Pa}, T$ the temperature in $\mathrm{K}, e$ the partial pressure of water vapor in $\mathrm{Pa}, N_{\mathrm{e}}$ the density of the free electrons in $\mathrm{m}^{-3}$ and $f$ the radar frequency in $\mathrm{Hz}$. The second term on the right side describes the dipole moments due to the polarization field of neutral molecules. The last two terms on the right side contribute only marginally to the refractive index and can be neglected in further calculations. Thus (11) simplifies to

$$
n=1+7.76 * 10^{-7} \frac{p_{\mathrm{a}}}{T}
$$

and the relative change of the electromagnetic refractive index can be calculated using

$$
\frac{\Delta n}{n}=7.76 * 10^{-7}\left(\frac{\Delta p}{T}-\frac{p_{\mathrm{a}}}{T^{2}} \Delta T\right) .
$$

Combining the adiabatic temperature gradient $\frac{\Delta T}{\Delta z}=\frac{-\rho}{c_{\mathrm{p}}}$ with the hydrostatic equation $\Delta p=$ $-\rho g \Delta z$, leads to $\Delta T=\frac{\Delta p}{\rho c_{\mathrm{p}}}$ [13]. Inserting this result into (13) and using $p=1.013 * 10^{5} \mathrm{~Pa}$, $T=273 \mathrm{~K}, \rho=1.2 \frac{\mathrm{kg}}{\mathrm{m}^{3}}$ and $c_{\mathrm{p}}=1005 \frac{\mathrm{J}}{\mathrm{kg} \mathrm{K}}$, the expression for refractive index simplifies to

$$
\frac{\Delta n}{n} \approx 2 * 10^{-9} \Delta p \text {. }
$$

The amplitude of the sound pressure variation for an isotropic radiator at a distance $r$ is given by [14]

$$
\Delta p=\sqrt{\frac{\rho c_{\mathrm{a}} P_{\mathrm{a}}}{4 \pi r^{2}}}
$$

where $P_{\mathrm{a}}$ is acoustic transmitted power in $\mathrm{W}$. Finally, (15) can be used in (14) and leads to

$$
\frac{\Delta n}{n} \approx 1.6 * 10^{-8} \frac{\sqrt{P_{\mathrm{a}}}}{r} \text {. }
$$

The change in the refractive index is thus inverse proportional to the distance $r$ and proportional to the square root of the acoustic transmission power $P_{\mathrm{a}}$.

\subsection{Receive power}

Marshall [15] and Clifford [16] describe in their work an expression for the receive power $P_{\mathrm{r}}$ of radar waves scattered at sound waves

$$
\begin{gathered}
P_{\mathrm{r}}=\left(\frac{\Delta n}{n}\right)^{2} \frac{4 \pi^{2} P_{\mathrm{t}} G_{\mathrm{t}} G_{\mathrm{r}}}{\lambda_{e}^{2} r^{2}}\left(\frac{\Delta r}{2}\right)^{2} . \\
\operatorname{sinc}^{2}\left[\left(2 k_{e}-k_{a}\right) \frac{\Delta r}{r}\right] .
\end{gathered}
$$

$P_{\mathrm{t}}$ describes the transmitted radar power in $\mathrm{W}$, $G_{\mathrm{t}}$ and $G_{\mathrm{r}}$ the gain of the transmitting respectively receiving antenna in $\mathrm{dBi}, k_{\mathrm{e}}=\frac{2 \pi}{\lambda_{\mathrm{e}}}$ and $k_{\mathrm{a}}=\frac{2 \pi}{\lambda_{\mathrm{a}}}$ the radar and acoustic wave number respectively and $r$ the range of the target in $\mathrm{m}$. So $r$ describes the current distance of the sound wave to the measurement system. If the radar is monostatic, then $G_{\mathrm{t}}=G_{\mathrm{r}} . \Delta r=$ $N_{\text {BRAGG }} \cdot \lambda_{\mathrm{a}}$ represents the length of the acoustic packet and can be calculated with the number of the acoustic cycles $N_{\text {BRAGG }}$ and the acoustic wavelength $\lambda_{\mathrm{a}}$. Combining the relative change in refractive index (16) with (17) leads to the RASS equation

$$
\begin{gathered}
P_{\mathrm{r}} \propto\left(\frac{\lambda_{\mathrm{a}}}{\lambda_{\mathrm{e}}}\right)^{2} \frac{P_{\mathrm{t}} G_{\mathrm{t}}^{2} P_{\mathrm{a}} N_{\mathrm{BRAGG}}^{2}}{r^{2}} . \\
\operatorname{sinc}^{2}\left[\left(2 k_{\mathrm{e}}-k_{\mathrm{a}}\right) \frac{\Delta r}{r}\right] .
\end{gathered}
$$

The RASS equation shows that the receive power will be proportional to $\mathrm{r}^{-2}$ instead of the traditional $r^{-4}$ for the classical radar equation [17]. The $r^{-2}$ dependence is due to the spherically divergent acoustic wave. This is a great benefit of the focused geometry [2].

\subsection{Bragg condition}

As already mentioned, the optimal Bragg condition is only valid if the radar wavelength is twice as large as the acoustic wavelength see (1). Substituting the Bragg condition into the RASS equation (18) maximizes the sinc function. This means that the backscattered electromagnetic power will be maximized. Due to the small change in the refractive index, or the low backscatter of the electromagnetic power on an acoustic wavefront, it is endeavored to hit the Bragg condition as well as possible. This leads to a constructive interference of the scattered electromagnetic power at the acoustic wavefronts in the propagation direction. In addition, the received power can be improved by increasing $N_{\text {BRAGG }}$. The received power is proportional to $N_{\mathrm{BRAGG}}^{2}$. Thus, doubling the Bragg cycles results in an increase in received power by $6 \mathrm{~dB}$. Therefore, a compromise must 
be found between the spatial resolution and sufficient signal-noise ratio. The importance of an optimal Bragg condition is shown with realworld measurement results in Section 5 .

\section{Realization, measurement setup}

There are several possible RASS configurations. The Radar can be monostatic or bistatic. In addition, the radar and the acoustic sources can be either continuous wave (CW) or pulsed [4]. In this work a monostatic CW radar with pulsed sound is presented. This configuration has the advantage that all previously emitted sound pulses have sufficient time to die away and thus there are no significant reflections in enclosed environments.

\subsection{Level plan}

The RASS system is designed to measure indoor room temperatures. For this purpose, a $10 \mathrm{GHz}$ synthesizer with $10 \mathrm{dBm}$ output power was chosen for the radar system.

Fig. 2 shows a block diagram of the setup of the RASS. To get the highest possible output power, the $1 \mathrm{~dB}$ compression point of the $23 \mathrm{~dB}$ amplifier in front of the circulator was approached. The total loss in the transmit path is $\approx 8 \mathrm{~dB}$, which includes the insertion losses, splitter loss, attenuator and cable loss. The antenna has a gain of $20 \mathrm{dBi}$ with a $-3 \mathrm{~dB}$ beam width of $14^{\circ}$. Thus, the equivalent isotropically radiated power (EIRP) is $43 \mathrm{dBm}$.

For 100 acoustic cycles $\left(N_{B R A G G}=100\right)$ the radar cross section (RCS) is $-60 \mathrm{dBm}^{2}$. A description as well as the calculation of the RCS is discussed by the authors in [18]. With the RASS equation (18) the path loss can be calculated. For this radar setup and a distance of $5 \mathrm{~m}$, the path loss is approximately $-90 \mathrm{~dB}$. Thus, the receive power is $-87 \mathrm{dBm}$. After the mixer, the low-frequency Doppler signal is again amplified by $20 \mathrm{~dB}$ with a programmable gain amplifier ( $50 \Omega$ output). A power of $-75 \mathrm{dBm}$ is digitalized at the analog-to-digital converter (ADC) with $1 \mathrm{~V}$ reference voltage. With 16 bit $A D C$ resolution, the signal can be detected.

Due to the Bragg condition and $10 \mathrm{GHz}$ radar frequency, wavelengths of $\lambda_{\mathrm{a}}=15 \mathrm{~mm}$ $\lambda_{\mathrm{e}}=30 \mathrm{~mm}$ result at room temperatures.

The right path of the block diagram shows an ultrasonic loudspeaker connected to an audio amplifier. The bandwidth of the tweeter is $4-25 \mathrm{kHz}$

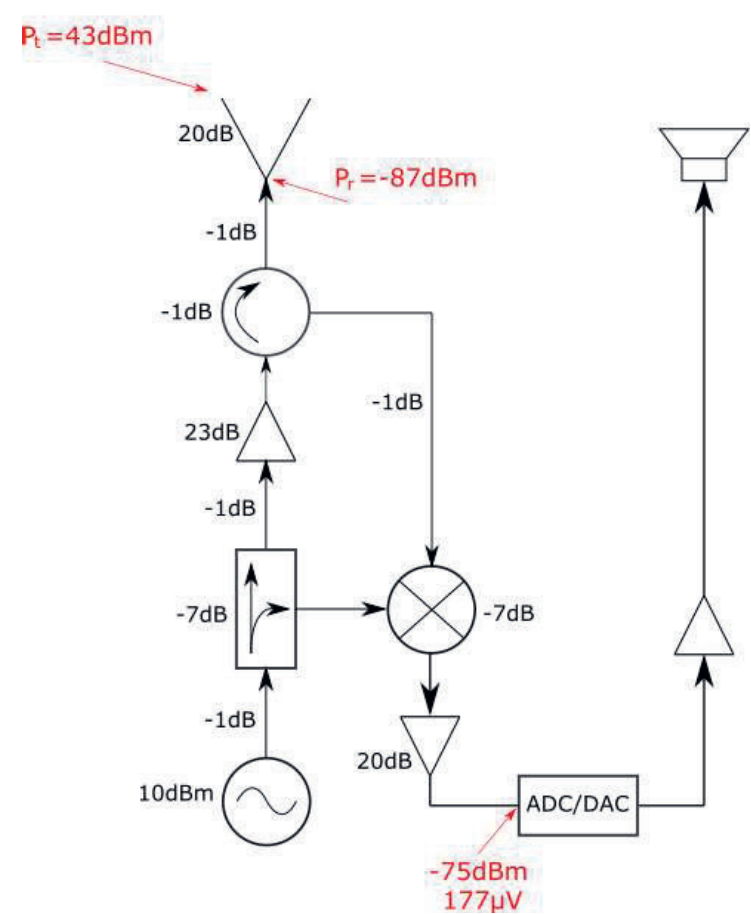

Figure 2: Level plan of the monostatic radar setup. $-75 \mathrm{dBm}$ input power at the ADC can be detected with a resolution of 16 bit and $1 \mathrm{~V}$ reference voltage.

\subsection{Collocation}

Another important prerequisite for RASS functionality is collocation. This means, that the phase center of the radar horn and the loudspeaker should be identical. As proposed by Weiss [4, 5], a fine metal grid can be used to enable this. Figure 3 shows the set-up of the measurement head.

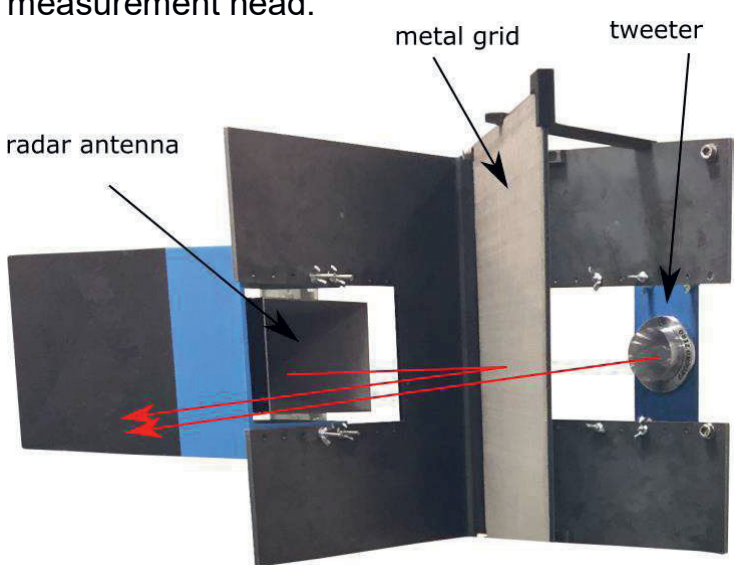

Figure 3: To get the highest possible reflectivity of the radar waves, the phase center of the radar horn and the loudspeaker should be identical, i.e. collocated. Utilizing a metal grid as proposed in [4] enables this.

The metal grid redirects the radar waves by $90^{\circ}$ and the sound waves travels straight through the grid. Thaller [19] shows in his work, that the grid has only a little influence of 
the sound propagation and can be neglected. If the phase centers are not virtually the same, then there is no optimum overlapping of the wavefronts and thus a lower receive power.

Influence of a horizontal wind on the receive power was theoretically studied by Peters et al. [20], Karyukin [21] and Kon [22]. A horizontal wind causes a virtual displacement of the sound source. The decrease of the receive power can be calculated by

$$
I=e^{\frac{-2 \rho_{v}^{2}}{D^{2}}}
$$

where $\rho_{v}$ is the virtual horizontal displacement of the sound source and $D$ is the $e^{-1}$ diameter of the radar antenna. Equation (19) is verified with real world measurement data in the next section.

\section{Measurement results}

As already mentioned, an optimal Bragg condition is essential to obtain sufficient receive power. The measurements were performed in a closed laboratory room. The room temperature was $25.8^{\circ} \mathrm{C}$ and was measured with a PT100 thermocouple type K. For different sound frequencies, which can be calculated by evaluation of

$$
f_{\mathrm{a}}=\frac{\sqrt{\frac{R_{\mathrm{u}} \kappa T}{\mu}}}{\lambda_{\mathrm{a}}}=1336.33 \cdot \sqrt{T},
$$

temperatures between $12^{\circ} \mathrm{C}$ and $38^{\circ} \mathrm{C}$ were chosen. Figure 4 shows the levels of electromagnetic backscattered power at different transmitted sound frequencies (temperatures). If the Bragg condition is matched exactly, maximum power will be backscattered. This is the case when the temperature of the transmitted frequency coincides with the actual temperature. For the measurement a sound packet length $\Delta \mathrm{r}$ of $1.5 \mathrm{~m}$ was chosen. With a threshold of $-6 \mathrm{~dB}$ a temperature range of $\approx 20-$ $32^{\circ} \mathrm{C}$ can be meaningful covered under these conditions. This corresponds to $\pm 6^{\circ} \mathrm{C}$ of the temperature to be measured. The longer the sound packet length (more Bragg cycles $N_{\text {BRAGG }}$ ), the smaller the temperature range that can be covered. This is discussed in detail in [1] and [18]. With this setup and settings, a signal-to-noise ratio (SNR) of $17 \mathrm{~dB}$ is possible with an optimal Bragg condition.

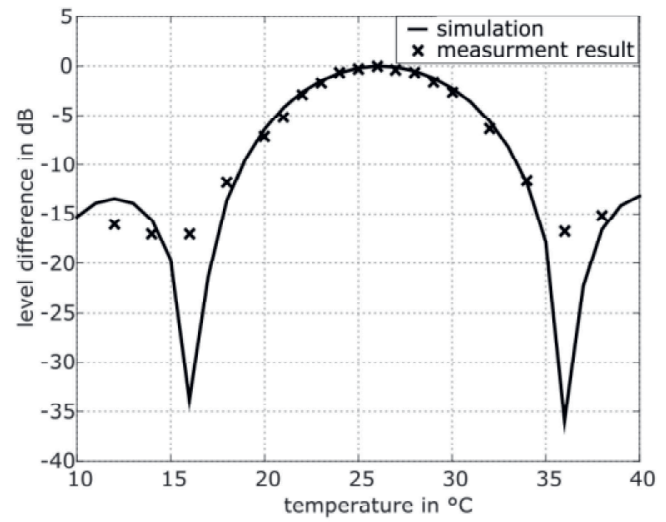

Figure 4: Comparison between the simulation and measurement result of the Bragg condition. Parameter: $N_{\mathrm{BRAGG}}=66, \lambda_{\mathrm{e}}=30 \mathrm{~mm}$, number of averaged measurements $=200$

To verify the collocation (Section 4.2), the speaker was moved in $5 \mathrm{~mm}$ steps. Figure 5 shows the measurement results compared to the simulation (19). If the collocation is not maintained exactly, there is an exponential loss in the received power. The measurement result shows a good agreement with the simulation.

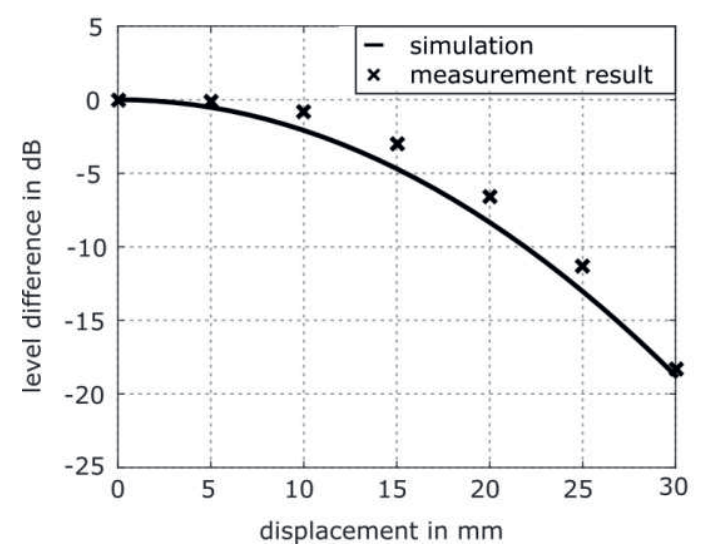

Figure 5: Horizontal displacement of the speaker in $5 \mathrm{~mm}$ steps. If the collocation is not maintained exactly, there is an exponential loss in the received power (19). Parameter: $N_{\text {BRAGG }}=66, \lambda_{\mathrm{a}}=15 \mathrm{~mm}, \lambda_{\mathrm{e}}=30 \mathrm{~mm}$, number of averaged measurements $=200$

\section{Conclusion and future work}

This article describes a radio-acousticsounding system, which can be used in industrial applications. The two most important conditions for an optimal Bragg reflection were shown and verified with real world measurement data.

It is important to characterize the limits of a system and the results presented in this paper are important steps for a successful and reliable assertion regarding the quality of a RASS measurement. 
For industrial applications, it is important to know the measurement uncertainty of a measuring system. The Cramer-Rao lower bound $[23,24]$ can be used to calculate the lower bound of the temperature variance. These accuracy limits, achievable resolution and methods of signal processing will be discussed in a future work.

\section{Acknowledgement}

The authors gratefully acknowledge the partial financial support for the work presented in this paper by the Austrian Research Promotion Agency (FFG) under contract grant 861570 and voestalpine Stahl $\mathrm{GmbH}$.

\section{Literatur}

[1] Stuart Bradley, "Atmospheric Acoustic Remote Sensing", CRC Press, ISBN 0-8493-3588-4, 2008.

[2] J. A Saffold, F. Williamson, K. Ahuja, L. Stein, M. Muller, "Radar-acoustic Interaction for IFF Applications", Radar Conference, Waltham USA, S. 198-202, 1999

[3] Benjamin Moussa Daas, "Die elektromagnetischakustische Wechselwirkung und ihre Anwendung zur berührungslosen Entfernungsund Temperaturmessung in geschlossenen Räumen", Shaker Verlag, Aachen, ISBN 38265-2148-X, 1996

[4] M. Weiß, R. Knöchel, "A Monostatic RadioAcoustic Sounding System Used as an Indoor Remote Temperature Profiler", IEEE Transaction on Instrumentation and Measurement, Vol. 50, No. 5, 2001

[5] M. Weiß, R. Knöchel, "Monostatic RadioAcoustic Sounding System", Geoscience and Remote Sensing Symposium, Hamburg, Germany, S. 29-31, 2001

[6] D. Exel, B. Zagar, S. Schuster, V. Ganglberger, J. Reisinger, "Parameter Estimation and Performance Bounds for Radiometric Model-based Non-contact Temperature Measurement', IEEE International and Measurement Technology Conference, Turin, Italien, S. 641-646, 2017

[7] D. Exel, B. Zagar, S. Schuster, V. Ganglberger, J. Reisinger, "Parameterschätzung und Cramér-Rao-Schranken bei der berührungslosen Temperaturmessung", tm-Technisches Messen, De Gruyter Oldenbourg, Vol. 86, S. 45-56, 2019

[8] D. Exel, B. Zagar, S. Schuster, V. Ganglberger, J. Reisinger, " Unsicherheitsbetrachtungen der Temperaturmessung aus Daten einer Multispektralkamera", Tagungsband der 5. Tagung Innovation Messtechnik, 2017

[9] R. P. Feynman, R. B. Leighton, M. Sands, "Feynman-Vorlesung über Physik 2 Strahlung und Wärme", De Gruyter, 6. Auflage, ISBN 978-3-11-036770-6, 2015
[10] F. Wächter, "Zur Gasanalyse durch Messung von Schallgeschwindigkeit und Wärmeleitfähigkeit', Proc. DAGA02, 2002, S. 386-387.

[11] D. Exel, S. Schuster, S. Scheiblhofer, D. Zankl, V. Ganglberger, J. Reisinger, B. Zagar, "Ein kompaktes Radio-Acoustic-Sounding System zur Temperatur- und Strömungsgeschwindigkeitsmessung", Tagungsband der 6 . Tagung Innovation Messtechnik, 2019

[12] B.B. Balsley, K.S. Gage, "The MST radar technique: Potential for middle atmospheric studies", Pure and Applied Geophysics, Vol. 118, S. $452-493,1980$

[13] F. Bernhard, "Handbuch der Technischen Temperaturmessung", Springer Vieweg, ISBN 978-3-642-24506-0, 2014

[14] J. R. Kutia, W. Xu, "A Portable Radio-Acoustic Sounding System for Temperature Profiling Within the Boundary Layer", Industiral Electronics Society, IECON, S. 2308-2313, 2014

[15] J. M. Marshall, A. M. Peterson, A. A. Barnes, "Combined Radar-Acoustic Sounding System", Applied Optics, Vol. 11, S. No.1, 108-112, 1972

[16] S.F. Clifford, T.I. Wang, "The range limitation on radar-acoustic sounding system (RASS) due to atmospheric refractive turbulence", IEEE Transactions on Antennas and Propagation, Vol. 25, S. 319-326, 1977

[17] M. Skolnik, "Introduction to Radar Systems", McGraw-Hill Book Company, ISBN 0-07057909-1, 1981

[18] D. Exel, S. Schuster, K. Thaller, B. Zagar, D. Zankl, S. Scheiblhofer, V. Ganglberger, J. Reisinger, "Radio-Acoustic-Sounding System ortsaufgelöste akustische Temperaturmessung im industriellen Umfeld", e\&i Elektronik und Informationstechnik, Vol. 135, No. 6, S. 389-395

[19] K. Thaller "Messaufbau zur interferometrischen Schallfeldmessung mit tomografischer Rekonstrunktion", Linz, 2008

[20] G. Peters, H. Timmermann, H. Hinzpeter, "Temperature sounding in the planetary boundary layer by RASS-system analysis and results", Int. J. Remote Sens., 4, S. 49-63, 1983

[21] G. A. Karyukin, "Influence of wind on operation of radar-acoustic atmospheric sounding system", Izv. Acad. Sci. USSR Atoms. Oceanic Phys., 18, S. 26-30, 1982

[22] A. I. Kon, "A bistatic radar-acoustic atmospheric-sounding system", Izv. Acad. Sci. USSR Atoms. Oceanic Phys., 17, S. 481-484, 1981, Innovation Messtechnik, Shaker Verlag $\mathrm{GmbH}$,

[23] S. Schuster, S. Scheiblhofer, A. Stelzer "DFT Frequency Estimation Threshold Level with Windowed Data", International Instrumentation and Measurement Technology Conference, Sorrento, Italy, 2006

[24] S. M. Kay "Fundermentals of Statistical Signal Processing-Estimation Theory", Englewood Cliffs, Upper Saddle River, NJ: Prentice Hall, 1993 